\title{
Neuroscience
}

\section{Flipping the switch on the thermoregulatory system}

\author{
Clifford B. Saper \& Natalia L. S. Machado
}

A population of excitatory neurons has been found to have a key role in controlling body temperature in rodents. The discovery adds to a body of work that is raising questions about long-standing models of thermoregulation. See p.109 \& p.115

Body temperature in mammals is tightly controlled ${ }^{1}$, and is typically maintained to within about $0.5^{\circ} \mathrm{C}$ of an animal's mean core temperature (usually around $37^{\circ} \mathrm{C}$ ) throughout life. However, lack of food can cause some mammals to enter a sleep-like state - called torpor or hibernation, depending on its duration - in which the body temperature can drop by $5-10^{\circ} \mathrm{C}$ (or, in some cases, even more) to conserve energy. Animals can also increase their body temperature (fever) in response to infection, slowing the replication of some invaders and improving the animal's chance of survival ${ }^{2}$. It has long been known that these regulatory feats are accomplished by neurons in the brain's preoptic area, but the exact identities of those neurons and their connections have not been understood. On pages 109 and 115, Takahashi et $a l_{.}{ }^{3}$ and Hrvatin et al. ${ }^{4}$ add to a flurry of papers that are revolutionizing our understanding of the preoptic neurons at the heart of thermoregulation.

Decades of research into thermoregulation have produced a model ${ }^{1}$ whereby excitatory neurons in a region of the preoptic area called the median preoptic nucleus are activated by warming of the skin. In the model, these excitatory neurons activate inhibitory

\section{“This model calls for reconsideration of much of what we thought we knew about thermoregulation."}

neurons in an adjacent brain region, the medial preoptic area. The inhibitory neurons express an enzyme called glutamic acid decarboxylase (GAD), which synthesizes the inhibitory neurotransmitter molecule GABA. The model posits that these neurons, which are

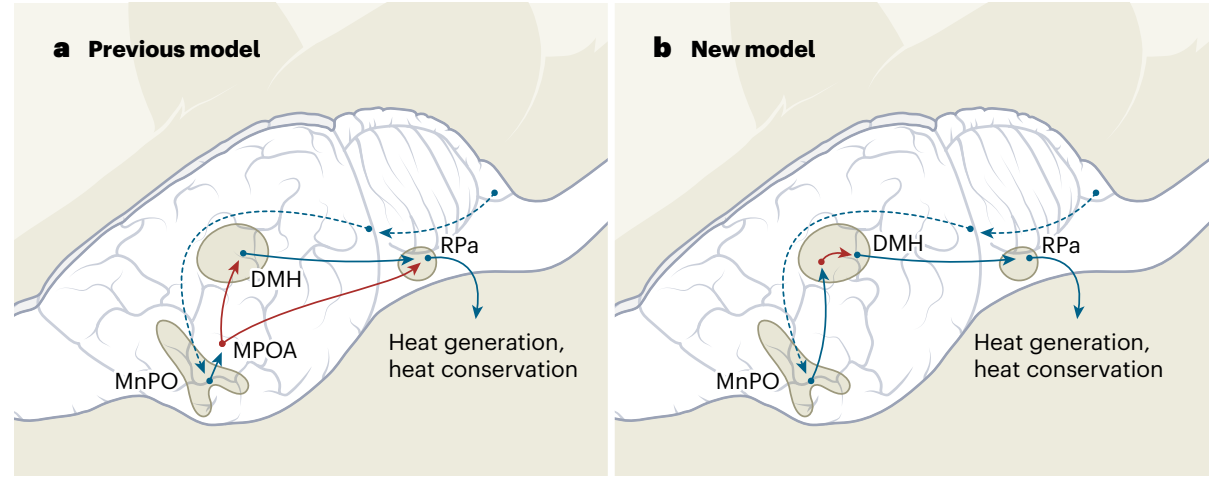

Figure 1 | Pathways for thermoregulation. Thermoregulation involves complex networks of excitatory and inhibitory neurons (blue and red dots, respectively, with their projections indicated by arrows). Here, these circuits are shown in the mouse brain. a, An existing model (much of which was based on work in rats) posits that warming of the skin leads (through neuronal pathways indicated by dashed arrows) to activation of excitatory neurons in a brain region called the median preoptic nucleus (MnPO). These neurons activate inhibitory neurons in the adjacent medial preoptic area (MPOA), which project to two more brain regions the dorsomedial hypothalamus (DMH) and the raphe pallidus (RPa) - that generate and conserve heat. Thus, skin warming would cause a regulated reduction in body temperature. b, Takahashi et al. ${ }^{3}$ and Hvratin et al. ${ }^{4}$ build on a flurry of work that supports an alternative model. The groups find that a population of mostly excitatory neurons in the MnPO causes profound hypothermia. Takahashi et al. show that these neurons express the protein fragment QRFP (not shown). The neurons connect directly to the DMH, where they presumably contact local inhibitory neurons to exert their effect on thermoregulation. presumably GABA-releasing (GABAergic), then project to other regions of the brain, where they inhibit neurons that promote heat production and conservation. Thus, activity of the GABAergic neurons results in cooling (Fig. 1a). In cool ambient temperatures, the preoptic GABAergic neurons would be inhibited, releasing constraints on heat production and conservation. And during an inflammatory illness, the lipid prostaglandin E2 is released and acts on EP3 receptor proteins in the neurons, causing their inhibition and so promoting responses involving fever ${ }^{5,6}$.

However, this model began to show cracks after a series of studies demonstrated that the preoptic neurons that cause cooling when activated express specific genetic markers, including several that encode certain protein fragments $^{7}$ and receptors ${ }^{6,8,9}$. Analysis of these markers revealed that the key neurons causing hypothermia are located in the median preoptic nucleus, but not in the medial preoptic area. These studies also found that the hypothermia-causing preoptic neurons release the excitatory molecule glutamate as their main neurotransmitter, rather than GABA. Like many other cells in the preoptic area, these neurons contain GAD, but they don't express the vesicular GABA transporter (Vgat) protein, which is needed to load GABA into synaptic vesicles, enabling the neurotransmitter to be released from the cell. Instead, the neurons express the vesicular glutamate transporter 2 (Vglut2) protein, making them glutamate-releasing (glutamatergic) and excitatory, rather than inhibitory ${ }^{10}$.

Against this background, Takahashi and colleagues describe a genetic marker in mice for a particularly potent subset of preoptic thermoregulatory neurons: a gene that encodes the protein fragment pyroglutamylated RF-amide peptide (QRFP). The authors genetically engineered mice such that neurons that express QRFP could be activated by the injection of a small molecule, clozapine $N$-oxide. This chemogenetic activation caused the animals to become immobile, and led to a fall in body temperature to about $23-24^{\circ} \mathrm{C}$. This hypothermia was associated with slowed heart rate and respiration, as well as reduced metabolic rate, similar to that seen in torpor or hibernation.

The authors next produced mice in which specific QRFP neurons or their projections (axon terminals) could be activated by laser light. A torpor-like state was produced when the authors used this optogenetic activation to stimulate either the QRFP cell bodies or their terminals in the dorsomedial hypothalamus. This brain area is known to send projections to a site in the brain's medulla, the raphe pallidus, which promotes increases in body temperature. Interestingly, the body temperature of animals that had QRFP-neuron-induced hypothermia was still regulated, but around 
a lower setpoint. This situation is typical of hibernation, and suggests that, when the QRFP neurons are actively lowering body temperature, other non-QRFP preoptic neurons could continue to regulate body temperature, but at a lower level.

Takahashi et al. showed that nearly $80 \%$ of the QRFP neurons expressed Vglut2 but not Vgat. By contrast, only about $7 \%$ expressed Vgat but not Vglut2, and around $13 \%$ expressed both. Deleting Vgat from the QRFP neurons slightly slowed the initial fall in body temperature caused by activating these cells, but body temperature reached a level comparable to that of control animals after six hours. Deleting Vglut2, by contrast, prevented a hibernation-like state. Thus, the hypothermia produced by QRFP neurons is predominantly mediated by glutamatergic transmission.

Previous research has indicated that many of the preoptic neurons that drive hypothermia express proteins called pituitary adenylate cyclase-activating peptide (PACAP) and brain-derived neurotrophic factor (BDNF) ${ }^{7}$. Takahashi and co-workers demonstrated that most preoptic QRFP-expressing neurons also expressed BDNF and PACAP. However, about $75 \%$ of the BDNF and PACAP neurons in the median preoptic nucleus did not express QRFP. Similarly, 75\% of the QRFP neurons expressed the EP3 receptor, but many EP3-expressing neurons did not express QRFP.

Hrvatin and co-workers used a different approach. The group analysed a marker of neuronal activity to determine the neuronal populations activated during torpor caused by 24 hours of food deprivation. The active neurons were distributed similarly to QRFP cells, and many of them expressed PACAP. Thus, the results of the two studies, taking very different approaches, reinforce one another.

Taken together, these observations suggest that there are several subpopulations of thermoregulatory neurons clustered together in the median preoptic nucleus, each distinguished by a unique pattern of gene expression. Among these, the QRFP group seems to be particularly important for producing deep hypothermia. This process is necessary when animals do not have sufficient food available to maintain their typical levels of metabolism and activity. At such times, animals can undergo daily torpor (brief periods when their body temperature might drop to $30^{\circ} \mathrm{C}$ or lower for a few hours, frequently seen in mice and rats) or hibernation (long, seasonal periods of deeper hypothermia, such as is seen in bears).

If similar groups of QRFP-expressing neurons are found in humans, they could represent a way to induce therapeutic hypothermia - for example, after heart attack or stroke, slowing down metabolic processes to help limit tissue damage. By contrast, during inflammatory illness, inhibition of QRFP glutamatergic neurons by the EP3 receptor might play a key part in producing fever ${ }^{6}$. Learning how to control these QRFP neurons could provide insight that will aid the development of new fever-reducing drugs.

The enormous range of body temperatures regulated by the QRFP neurons suggests that they and the other subpopulations of thermoregulatory neurons in the median preoptic nucleus might be the centrepiece of the brain's thermoregulatory system. But if these neurons are excitatory, and if they act on neurons that cause heat generation and conservation, then there must be an inhibitory link, almost certainly consisting of local inhibitory neurons called interneurons (Fig. 1b). This model calls for reconsideration of much of what we thought we knew about thermoregulation, in particular the physiological roles of genetically distinct subpopulations of median preoptic thermoregulatory neurons.
Clifford B. Saper and Natalia L. S. Machado are in the Department of Neurology,

Division of Sleep Medicine, and Program in Neuroscience, Harvard Medical School, and Beth Israel Deaconess Medical Center, Boston, Massachusetts 02215, USA.

e-mail: csaper@bidmc.harvard.edu

\section{Atomic forces mapped out by lasers}

\section{Michael A. Sentef \\ The forces between electrons and nuclei in solids are difficult to image directly. A study shows that these forces can instead be indirectly imaged using the light emitted when the electrons are subjected to a strong laser field. See p.55}

One of the central goals of physics is to gain a detailed understanding of nature's building blocks and the mutual forces between them. In materials, such building blocks are atomic nuclei and the electrons that zip around between these nuclei, with forces acting on atomic length scales. Direct imaging of such forces using light is notoriously difficult and typically requires X-ray wavelengths. However, on page 55 , Lakhotia et al. ${ }^{1}$ demonstrate that indirect imaging is possible using visible light, even though the wavelengths of this light are about 10,000 times larger than atomic scales.

The authors achieved this feat using a method called high-harmonic generation, in which a strong laser field provides the electrons with more energy than they need to overcome the forces pulling them back to the nuclei. The shaken electrons then emit light at multiples of the laser frequency, known as high harmonics. This emission is a consequence of the nonlinearity of the energy 'landscape' that the electrons are subjected to inside the periodic lattice of nuclei when they are driven by an intense laser field.
To understand this effect, consider playing a note on a trumpet. When the instrument is played at normal strength, a pure tone is heard at the intended frequency. However, when one blows the trumpet strongly, higher overtones emerge because the amplitude of the instrument's excitation is sufficiently large to probe a nonlinear regime.

Electrons in solids are quantum-mechanical objects described by a wavefunction that determines the probability of finding them at a specific position and with a particular velocity or momentum. For free particles, momentum is the product of mass and velocity. However, electrons in solids are not free, but are affected by the potential energy provided by the uniform atomic lattice. The electrical forces applied to the electrons by the nuclei are given by the slope of the potential-energy landscape at each position (Fig. 1a) and are analogous to the gravitational forces pulling back a hiker in the mountains. But how can these forces be mapped out by shaking the electrons with a laser?

The answer to this question is best 
News \& views

Clarification

The caption for Figure 1 has been amended to make clear that mouse brains are depicted in the figure, and that much of the work leading to the previous model was performed in rats. 